\title{
Estimación de la densidad de madera en árboles de comunidades forestales templadas del norte del estado de Durango, México
}

\section{Estimating bole wood specific gravity in trees of temperate forest communities of northern Durango, Mexico}

\section{Flor María Silva-Arredondo ${ }^{1}$ y José de Jesús Návar-Cháidez}

\begin{abstract}
RESUMEN
El objetivo de esta investigación fue el de estimar la densidad básica de la madera y su nivel de variación con la altura y el diámetro en árboles de varias especies de los géneros Pinus y Quercus del norte del estado de Durango, México. Para esto se tomaron 843 especímenes a diferentes alturas del fuste y en diferentes secciones diamétricas de un muestreo aleatorio en 113 árboles. Se elaboraron probetas con dimensiones de $2,5 \mathrm{~cm} \times 2,5 \mathrm{~cm} \times 2,5 \mathrm{~cm}$. Los resultados muestran que la densidad básica es estadísticamente diferente entre los componentes de albura y duramen $(P>F=0,0001)$ y la altura relativa $(P>F=0,0001)$ del fuste en el género Pinus. Para el género Quercus no se encontraron diferencias estadísticamente significativas con los componentes de la madera o con la altura relativa. Los cálculos matemáticos indican que la densidad básica se debe de estimar a una altura dada por $0,22 \mathrm{H}$ y no como convencionalmente se hace a $1,3 \mathrm{~m}$.
\end{abstract}

PALABRAS CLAVE:

Albura y duramen, altura relativa, densidad de madera, nivel de variación.

\begin{abstract}
The objective of this research was to estimate basic wood specific gravity and its level of variation among biomass components of trees of several species of the genera Pinus and Quercus of northern Durango, Mexico. A total of 843 wood core sepecimens were taken at different heights and at different diameter sections from 113 trees. Fresh wood blocks of $2,5 \mathrm{~cm} \times 2,5 \mathrm{~cm} \times 2,5 \mathrm{~cm}$ were measured and once they were oven dried they were weighted to estimate the wood specific gravity. Results showed that the basic wood specific gravity was statistically different between biomass components along the diameter and height gradients $(P>F=0,0001)$ for trees of the genus Pinus unlike for trees of the genus Quercus. Mathematical evaluations show that wood cores for measuring the bole wood specific gravity must be taken at a height of $0,22 \mathrm{H}$ instead of the traditional height of $1,3 \mathrm{~m}$ at which it is conventionally evaluated.
\end{abstract}

KEY WORDS:

Sapwood and heartwood, relative height, wood density, level of variation.

1 Consultora Forestal. Linares, N.L., México.

2* Profesor-Investigador de Manejo de Recursos Naturales. CIIDIR-IPN Unidad Durango. Sigma núm. 119. Fracc. 20 de Noviembre II. Durango, Dgo. 34220. México. c.e.: jnavar@ipn.mx. Autor para correspondencia. 


\section{INTRODUCCIÓN}

La densidad básica (peso seco entre volumen húmedo) es un variable intrínseca de la madera que describe la cuarta dimensión del arbolado (Návar, 2010). Esta variable se emplea para estimar la biomasa de árboles y rodales forestales por medio de ecuaciones físicas o empíricas (Mohren y Klein Goldewijkt, 1994; Chavé et al., 2005; Návar, 2011). Es también necesaria para estimar los contenidos de varios elementos biogeoquímicos que se encuentran en las plantas (Chavé, 2002; Návar, 2009a, 2009b). La densidad básica se considera una característica de importancia económica, ya que determina en gran medida la calidad del producto final que se obtenga del árbol (Zobel y Van Buijtenen, 1989). Además, la productividad total de un rodal que es una variable que se encuentra en función de la biomasa de un árbol o de un rodal puede determinarse cuando se conoce la densidad promedio de la madera (Jenkins et al., 2001; Zobel y Talbert, 1988).

Por otro lado, la densidad de la madera varía dentro de la planta, durante la vida de la planta y entre individuos de una misma especie (Chavé, 2002; Daniel et al., 1982; Hocker, 1984; Parolin, 2002; Zobel y Talbert, 1988; Zobel y Van Buijtenen, 1989; Van Buijtenen, 1963). Varios factores del sitio, específicamente la cantidad de humedad presente en forma de precipitación o de cantidad de agua del suelo, parecen explicar en parte la variación tan amplia que presentan los individuos de una misma especie (Parolin, 2002).

La densidad básica varía también a lo largo del fuste y de la corteza hacia el centro del fuste. Esto sucede porque la parte distal de los árboles está compuesta por madera relativamente nueva, del año presente o anterior, mien- tras que en la parte basal del fuste la proporción de madera nueva es relativamente baja y la madera presente contiene un rango de edades anuales desde el nacimiento del árbol hasta el presente. Pocos trabajos han descrito la función de la densidad básica con la altura relativa del árbol. De la periferia al centro del fuste se encuentran: la corteza, la albura y el duramen. Estos componentes poseen también diferentes valores de densidad básica (Fearnside, 1997; Parolin, 2002; Návar et al., 2005). Una fuente importante de datos fue publicada recientemente sobre la densidad de la corteza y de la madera para árboles de Norteamérica por Miles y Smith (2009) que refleja en términos generales que la densidad básica es similar en la madera y en la corteza, pero que al nivel del árbol individual o de la especie se notan variaciones importantes. A pesar de estas diferencias, el método estándar para tomar muestras de madera para la medición de la densidad básica es a 1,30 m de la altura del árbol. Esto es, la toma de muestras se hace convencionalmente al diámetro a la altura del pecho (DAP) y corre el riesgo de sesgar la estimación de la cantidad de la biomasa y por consiguiente de otros parámetros derivados, tales como la biomasa, el contenido y los flujos de varios elementos bio-geoquímicos o la productividad de la especie o del rodal.

Como consecuencia, los objetivos del presente estudio fueron: a) determinar y b) modelar el comportamiento de la densidad básica a diferentes alturas relativas del fuste en la albura y el duramen de las principales especies de Pinus y Quercus del norte del estado de Durango, México. La hipótesis intrínseca que se plantea es que no existen variaciones de esta variable con la altura relativa del árbol, así como tampoco entre los componentes albura y duramen $y$, finalmente, que la densidad promedio de un árbol se puede estimar utilizando muestras de un 
punto del fuste a 1,30 m. Las especies de pino estudiadas fueron $P$. arizonica Engelmann, $P$. durangensis Martínez, $P$. engelmannii Carriere, $P$. teocote Schiede ex Schelchdental et Chamisso, P.cooperi Blanco y $P$. leiophylla Schiede ex Schelchdental et Chamisso. Las especies de encino muestreadas, de donde se colectaron las probetas fueron: $Q$. sideroxylla Humb \& Bonpl. y Q. crassifolia Humb \& Bonpl.

\section{METODOLOGÍA}

\section{Área de estudio}

El presente trabajo se realizó en las comunidades forestales El Tarahumar, El Tule y La Soledad, que se ubican en la Sierra Madre Occidental en los municipios de Tepehuanes y Guanaceví del estado de Durango. Se localizan específicamente al noroeste de la cabecera municipal de Tepehuanes y al oeste de la cabecera municipal de Guanacevi, dentro de las coordenadas de $25^{\circ} 33^{\prime} 48^{\prime \prime}$ a $25^{\circ} 49^{\prime} 07^{\prime \prime}$ latitud Norte y de $106^{\circ} 11^{\prime} 04^{\prime \prime}$ a $106^{\circ}$ $37^{\prime} 22^{\prime \prime}$ de longitud oeste con respecto al meridiano de Grenwich (Fig.1). Los tipos de vegetación presentes en la zona corresponden a bosques de pino, de pinoencino, de encino y otras asociaciones de pino-otras coníferas y pino-otras hojosas, donde los bosques más importantes en el área son del género Pinus, con una amplia distribución, encontrándose la mayor presencia de las especies Pinus arizonica, Pinus teocote y Pinus durangensis, siendo posible encontrar manchones puros de encino cuyas especies presentes son $Q$. crasifolia, Q. rugosa, Q. sideroxylla, etcétera.

La región se encuentra dentro de la provincia fisiográfica denominada Sierra Madre Occidental en la Subprovincia Sierra y Cañones Duranguenses. El clima que prevalece en el área de estudio es templado frío húmedo, $\mathrm{C}(\mathrm{w} 0)$ y $\mathrm{C}(\mathrm{w} 2)$, con una temperatura media anual de $10,5^{\circ} \mathrm{C}$ y una precipitación media anual de $932 \mathrm{~mm}$, con lluvias en verano. En general, los tipos de suelos que se encuentran en la región son los Litosoles, aunque también existen con menor frecuencia los Regosoles y los Feozems (INEGI, 2002).

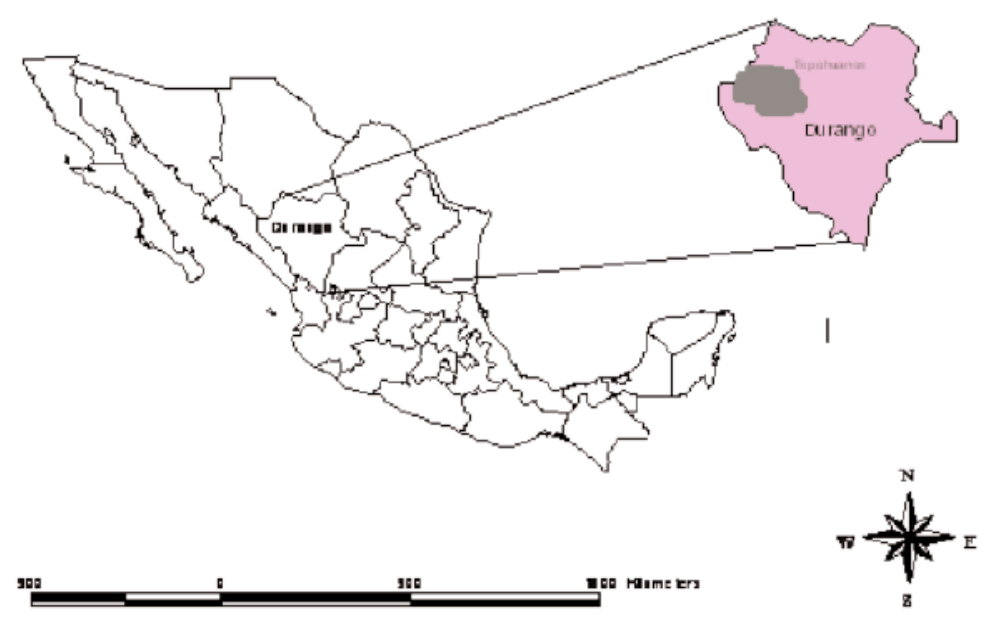

Figura 1. Localización del área de estudio en el noroeste de Durango, México. 


\section{Selección de los árboles muestra}

Se establecieron tres sitios de muestreo en las localidades conocidas como El Tule, La Soledad y Tarahumar, en los cuales se seleccionaron 66,34 y 13 individuos, respectivamente, cubriendo todas las categorías presentes en las diferentes áreas de corta de los aprovechamientos autorizados en los programas de manejo forestal vigentes de los servicios técnicos forestales. La variabilidad en el tamaño de muestra obedece al número de especies presentes en cada uno de los sitios y las categorías diamétricas existentes en las áreas de corta.

\section{Preparación de las probetas}

En cada árbol muestreado o derribado, el fuste se cortó en secciones de 2,5 $\mathrm{m}$ y se tomaron rodajas de cada sección a partir del tocón hasta la punta del árbol, registrándose el nombre de la especie, número de árbol, peso verde y altura de la sección (Figura 2). Posteriormente, se trasladaron al laboratorio y de cada rodaja se obtuvo una sección transversal de $6 \mathrm{~cm}$ de ancho y el largo en función del diámetro de la muestra. De esta muestra se tomó una probeta de $2.5 \times 2.5 \times 2.5 \mathrm{~cm}$ de la albura y otra del duramen (Fig. 3), las cuales se pesaron y se midieron en estado verde, llevándose a la estufa de secado a una temperatura constante de $105^{\circ} \mathrm{C}$ hasta obtener un peso constante determinando su peso seco y volviéndose a medir (Tabla 1). En total se colectaron 843 probetas, de las cuales 758 y 85 correspondieron a los géneros Pinus y Quercus, respectivamente, y de las cuales 451 y 392 a la albura y el duramen, respectivamente.

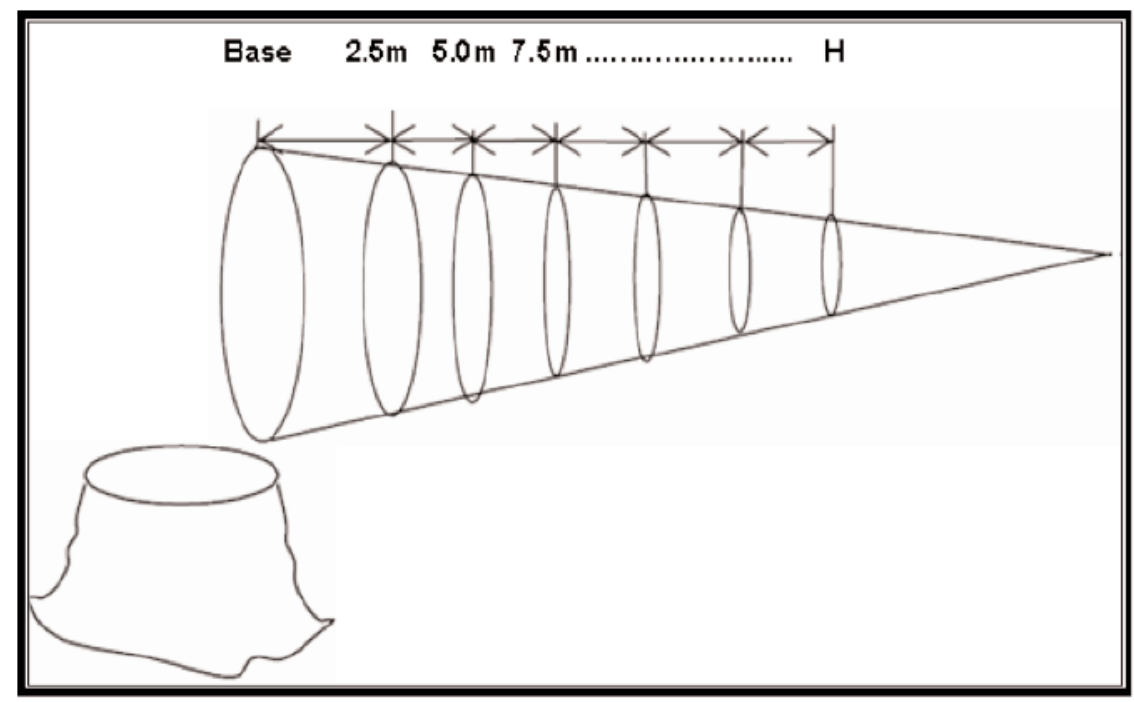

Figura 2. Rodajas tomadas a cada 2,5 m de las secciones del árbol para la obtención de las probetas. 


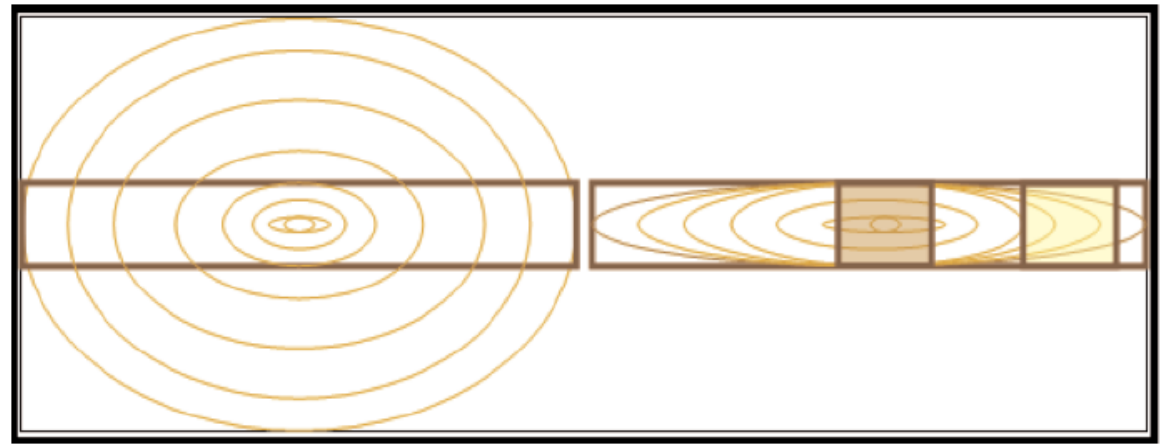

Figura 3. Obtención de probetas a lo largo de una sección del fuste.

Tabla 1. Lugar de procedencia, número de individuos y probetas por componente de los árboles analizados.

\begin{tabular}{|c|c|c|c|c|}
\hline Predio & Género & Núm. individuos & Componente & $\begin{array}{c}\text { Probetas } \\
\text { analizadas }\end{array}$ \\
\hline \multirow[t]{2}{*}{ El Tule } & Pinus spp & 36 & $\begin{array}{c}\text { Duramen } \\
\text { Albura }\end{array}$ & $\begin{array}{l}217 \\
193\end{array}$ \\
\hline & Quercus spp & 3 & $\begin{array}{l}\text { Duramen } \\
\text { Albura }\end{array}$ & $\begin{array}{c}12 \\
8\end{array}$ \\
\hline \multirow[t]{2}{*}{ La Soledad } & Pinus spp & 29 & $\begin{array}{c}\text { Duramen } \\
\text { Albura }\end{array}$ & $\begin{array}{l}132 \\
114\end{array}$ \\
\hline & Quercus spp & 5 & $\begin{array}{c}\text { Duramen } \\
\text { Albura }\end{array}$ & $\begin{array}{l}23 \\
19\end{array}$ \\
\hline \multirow[t]{2}{*}{ El Tarahumar } & Pinus spp & 10 & $\begin{array}{c}\text { Duramen } \\
\text { Albura }\end{array}$ & $\begin{array}{l}55 \\
47\end{array}$ \\
\hline & Quercus spp & 3 & $\begin{array}{c}\text { Duramen } \\
\text { Albura }\end{array}$ & $\begin{array}{l}12 \\
11\end{array}$ \\
\hline
\end{tabular}

\section{Procedimiento estadístico}

A cada probeta obtenida de la albura y el duramen se le determinó la densidad básica, $\rho$, de la siguiente forma: $\rho=$ peso anhidro/volumen verde. Con los datos de densidad básica se realizó un análisis de varianza para identificar diferencias con la altura relativa del fuste y con los componentes albura y duramen. Se utilizó el paquete estadístico SAS para correr el análisis estadístico y para el ajuste de las regresiones conducentes. El modelo lineal se ajusta bien a la relación de la densidad básica promedio con la altura relativa del árbol, como sigue: 
donde

$$
\rho=\alpha^{\prime}+\beta(h / H)
$$

$\alpha$ : parámetro que representa la intercepta de la relación $r$ contra $(\mathrm{h} / \mathrm{H})$ y que se interpreta como la densidad básica a la base del fuste.

$\beta$ : parámetro que representa la pendiente de la relación $r$ contra $(h / H)$ y que representa la tasa de cambio en la densidad básica en función de la altura relativa del árbol.

h: altura i del fuste

$\mathrm{H}$ : altura total del fuste.

Los parámetros estadísticos $\alpha$ y $\beta$ fueron estimados por medio de la técnica de cuadrados mínimos en regresión lineal, expresada en Návar (2009c). El punto de la altura en el cual se debe de tomar la muestra para obtener una probeta y medir la densidad básica promedio se determina, de acuerdo con Návar (20011), por medio de la ecuación siguiente:

$$
\begin{gathered}
E(X)=\int_{h / H^{=0}}^{h / H^{=1}}[\alpha+\beta(h / H)](h / H) \delta(h / H) \\
E(X)=\bar{X}=\int_{h / H^{=0}}^{h / H^{=1}}[\alpha+\beta(X)](X) \delta X \\
\int_{0}^{1.0} \alpha X+\beta X^{2} \delta X \\
\frac{\alpha}{2} X^{2}+\frac{\beta}{3} X^{3}
\end{gathered}
$$

donde

$\delta$ : $\quad$ Densidad básica promedio.

$\alpha$ y $\beta$ : Parámetros estadísticos a estimar.

$\mathrm{h} / \mathrm{H}$ : Altura relativa del árbol.

La desviación estándar y el intervalo de confianza de la longitud de la troza, donde se puede evaluar el intervalo de la altura, de donde se puede determinar la densidad básica de la madera, se estimaron, de acuerdo con Návar (2011) de la siguiente manera:

$$
\begin{array}{r}
S(X)=S=\sqrt{\mathrm{E}\left(X^{2}\right)-[E(X)]^{2}} \\
E\left(X^{2}\right)=\int_{x=0}^{x=1} X^{2}(\alpha+\beta X) \delta X \\
=\int_{x=0}^{x=1} \alpha x^{2}+\beta x^{3} \delta x \\
=\frac{\alpha}{3} x^{3}+\frac{\beta}{4} x^{4} \\
\sqrt{\left[\frac{\alpha}{3} x^{3}+\frac{\beta}{4} x^{4}\right]-\left[\frac{\alpha}{2} x^{2}+\frac{\beta}{3} x^{3}\right]^{2}}
\end{array}
$$

donde

S: Desviación estándar.

$\mathrm{X}$ : Altura relativa.

$\alpha$ y $\beta$ : Parámetros estadísticos a estimar.

El intervalo de confianza se estima de acuerdo con la ecuación siguiente:

$$
\text { I.C. }=X \pm \frac{S D}{\sqrt{n}} \cdot t_{\alpha, n-2}
$$

donde

I.C.: Intervalo de confianza.

$\alpha: \quad 0,05$.

SD: Desviación estándar,

$\mathrm{n}$ : Número de datos

\section{RESULTADOS Y DISCUSIÓN}

El valor promedio encontrado en la densidad básica del género Pinus fue de $0,47 \mathrm{~g} \mathrm{~cm}^{-3}$ con valores mínimo y máximo de $0,35 \mathrm{~g} \mathrm{~cm}^{-3}$ y $0,59 \mathrm{~g} \mathrm{~cm}^{-3}$, por lo que puede considerarse, de acuerdo con Echenique y Plumptre (1994), como una madera medianamente pesada (Tabla 2). El valor promedio estimado en este género parece ser ligeramente menor al calculado por López y Valencia (2001) para Pinus 
greggii en el norte de México, quienes encontraron valores de entre $0,40 \mathrm{~g} \mathrm{~cm}^{-3} \mathrm{y}$ $0,58 \mathrm{~g} \mathrm{~cm}^{-3}$ y mayor a los valores promedio registrados por Pérez et al. (2000) en Pinus taeda, quienes determinaron un valor promedio de $0,38 \mathrm{~g} \mathrm{~cm}^{-3}$, con valores mínimo y máximo de $0,29 \mathrm{~g} \mathrm{~cm}^{-3}$ a $0,58 \mathrm{~g} \mathrm{~cm}^{-3}$.

La densidad básica promedio varió entre las especies estudiadas (Fig. 4). Aunque las variaciones más notorias fueron entre los géneros de pino $(0,47 \pm 0,05)$ y encino $(0,77 \pm 0,02)$, también se registraron diferencias estadísticamente significativas entre las especies del género Pinus. $P$. teocote $(0,52 \pm 0,07)$ registró valores mayores que $P$. arizonica $(0,42 \pm 0,03)$.

Para los componentes de la madera, para el género pino, el duramen fue diferente también entre las especies $P$. teocote $(0,57 \pm 0,10)$ y $P$. arizonica $(0,39 \pm 0,028)$. En la albura, $P$. durangensis $(0,53 \pm 0,05)$ registró mayores valores que $P$. cooperi $(0,44 \pm 0,025)$.
Las variaciones tan amplias en la desviación estándar fueron del orden de $0,05 \mathrm{~g} \mathrm{~cm}^{-3}$; con desviaciones mínimas y máximas de 0,032 y $0,085 \mathrm{~g} \mathrm{~cm}^{-3}$, respectivamente. Considerando que esta variable se distribuye normalmente, $68 \%$ de la densidad básica que se ha publicado para estos pinos tendrá valores de $0,47 \mathrm{~g} \mathrm{~cm}^{-3} \pm 0,05 \mathrm{~g} \mathrm{~cm}^{-3}$. Se ha discutido que esta variable se encuentra en función de las características del sitio tales como: contenido de humedad del suelo, productividad, densidad o grado de densidad del arbolado, etc. (Parolin, 2002). Sin embargo, estas variables no fueron medidas cuando se cosecharon los árboles para este estudio y como consecuencia tampoco se puede especular más sobre la dependencia de esta variable con aquellas no medidas.

El valor promedio encontrado en la densidad básica del género Quercus fue de $0,77 \mathrm{~g} \mathrm{~cm}^{-3}$ con valores mínimos y máximo de $0,61 \mathrm{~g} \mathrm{~cm}^{-3}$ a $0,86 \mathrm{~g} \mathrm{~cm}^{-3}$, por

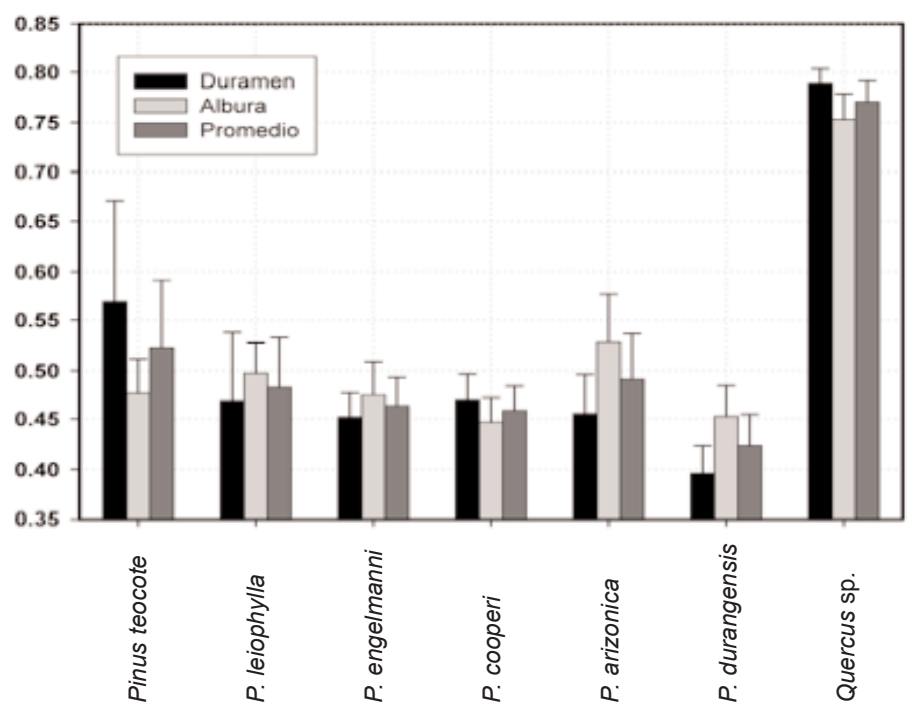

Figura 4. Los estadísticos de la densidad básica para las especies estudiadas en el noroeste del Estado de Durango, México. 
lo que puede considerarse, de acuerdo con Echenique y Plumptre (1994) como una madera altamente pesada (Tabla 2). El valor promedio estimado de la densidad de la madera en este género es mayor al registrado por Nájera et al. (2005) para Quercus laeta de la región centro sur de la Sierra Madre Occidental del estado de Durango, México. Estos investigadores midieron un promedio de $0,68 \mathrm{~g} \mathrm{~cm}^{-3}$. Los estadísticos estimados para esta especie se encuentran dentro del rango de valores mencionados en la literatura de la clasificación de la madera de acuerdo con su densidad (Echenique y Díaz, 1972).

\section{Diferencias de la densidad básica entre la albura y duramen}

La densidad básica resultó ser estadísticamente igual cuando se contrastaron los componentes albura y duramen, en los géneros Pinus ( $P \geq F=0,5409$ ) y Quercus $(P \geq F=0,0526)$. Es decir, las probetas se pueden obtener en estos géneros independientemente del componente de la madera, en la albura o el duramen. Para esto se recomienda seguir con las instrucciones descritas por Chavé (2002). La densidad tampoco varió estadísticamente entre los componentes albura y duramen y la altura relativa de la troza en el género Quercus $(P>F=0,2243)$. Esto concuerda con el reporte de Pearson y Williams (1952) y Parolin (2002), de que las variaciones entre algunas especies de los árboles son más significativas que la variación dentro del mismo árbol.

Sin embargo, la densidad básica del duramen cambia con la altura relativa en las especies de pino estudiadas $(P>F=0,0001)$ y se ajusta la tendencia a una línea recta con pendiente negativa $\left(r^{2}=0,30\right.$ y $\left.S_{x}=0,06\right)$. Es decir, la densidad básica disminuye conforme aumenta la altura relativa del árbol. Por ejemplo, esta adquiere un valor de $0,46 \mathrm{~g} \mathrm{~cm}^{-3}$ cuando la altura relativa es de $10 \%$ y disminuye hasta $0,41 \mathrm{~g} \mathrm{~cm}^{-3}$ cuando la altura relativa del árbol es $100 \%$. Por ejemplo, en un árbol que mide $13 \mathrm{~m}$ de alto, existe una reducción de $10 \%$ en la densidad cuando la probeta se toma a 1,3 m, que en contraste a cuando se toma a $11,7 \mathrm{~m}$. Esta variación hace que un fuste con $1 \mathrm{~m}^{3}$ de madera posea o $460 \mathrm{~kg}$ o $410 \mathrm{~kg}$, con una diferencia de $50 \mathrm{~kg}$ que cuando se multiplica por los árboles que componen un bosque esta diferencia aumente notoriamente.

La relación entre la densidad básica promedio de la madera y la altura relativa para el duramen de especies del género

Tabla 2. Densidad básica promedio para los géneros Pinus y Quercus en el Norte de Durango, México.

\begin{tabular}{lccccc}
\hline Género & $\begin{array}{c}\text { Valor máximo } \\
\left(\mathrm{g} \mathrm{cm}^{-3}\right)\end{array}$ & $\begin{array}{c}\text { Valor mínimo } \\
\left(\mathrm{g} \mathrm{cm}^{-3}\right)\end{array}$ & $\begin{array}{c}\text { Promedio } \\
\left(\mathrm{g} \mathrm{cm}^{-3}\right)\end{array}$ & $\begin{array}{c}D S \\
\left(\mathrm{~g} \mathrm{~cm}^{-3}\right)\end{array}$ & $\begin{array}{c}\text { IC } \\
\left(\mathrm{g} \mathrm{cm}^{-3}\right)\end{array}$ \\
\hline Pinus & 0,52 & 0,40 & 0,47 & 0,05 & 0,05 \\
Quercus & 0,86 & 0,61 & 0,77 & 0,11 & 0,02 \\
\hline
\end{tabular}

donde: $\mathrm{g} \mathrm{cm}^{-3}=$ gramos sobre centímetros cúbicos; $\mathrm{DS}=$ desviación estándar; $\mathrm{IC}=$ intervalo de confianza. 
Pinus ( $P$. arizonica, $P$. durangensis, $P$. engelmannii, $P$. teocote, P.cooperi y $P$. leiophylla) del norte del estado de Durango, México, se presenta en la figura 5. La ecuación que describe la línea recta de la tendencia, cuando se integra para estimar el promedio, la desviación estándar e intervalo de confianza resultan en: $0,222 \mathrm{H} ; 0,044 \mathrm{H}$ y $0,015 \mathrm{H}$, respectivamente. Esta información indica que la densidad básica del duramen se puede determinar a alturas relativas con un intervalo de $0,222 \mathrm{H} \pm 0,015 \mathrm{H}$. Es decir, para árboles de $10 \mathrm{~m}, 15 \mathrm{~m}, 20 \mathrm{~m}$ o $25 \mathrm{~m}$ de altura, la probeta debe de tomarse a $2,2(0,15) ; 3,3(0,22) ; 4,4(0,30) \circ 5,5$ $(0,37)$ metros, respectivamente. Solo cuando los árboles tienen una altura de 6 $\mathrm{m}$, la probeta puede tomarse a 1,3 m.

La relación entre la densidad básica promedio de la madera y la altura relativa para la albura de especies del género Pinus ( $P$. arizonica, $P$. durangensis, $P$. engelmannii, $P$. teocote, P.cooperi $y$ $P$. leiophylla) del norte del estado de
Durango, México se presenta en la figura 6. La densidad básica de la albura cambia con la altura relativa en las especies de pino estudiadas $(P>F=0,0001)$ y se ajusta la tendencia a una línea recta con pendiente negativa $\left(r^{2}=0,36\right.$ y $\left.S_{x}=0,06\right)$. En árboles de $13 \mathrm{~m}$ de altura, la densidad básica de la albura cambia desde $0.493 \mathrm{~g} \mathrm{~cm}^{-3}$ cuando la probeta se toma a 1,3 m hasta $0.433 \mathrm{~g} \mathrm{~cm}^{-3}$ cuando la probeta se toma a $11,7 \mathrm{~m}$. Es decir, existe una reducción de $12 \%$ en el valor de la densidad básica cuando se toma a $11,7 \mathrm{~m}$ con respecto a aquella medida a 1,3 $\mathrm{m}$.

La ecuación que describe la línea recta de la tendencia, cuando se integra para estimar el promedio, la desviación estándar e intervalo de confianza resultan en: $0,225 \mathrm{H} ; 0,043 \mathrm{H}$ y $0,015 \mathrm{H}$, respectivamente. Esta información indica que la densidad básica de la albura se puede determinar a alturas relativas con un intervalo de $0,225 \mathrm{H} \pm 0,015 \mathrm{H}$. Es decir, para árboles de $10 \mathrm{~m}, 15 \mathrm{~m}, 20 \mathrm{~m}$ o $25 \mathrm{~m}$ de altura, la probeta debe de colectarse a

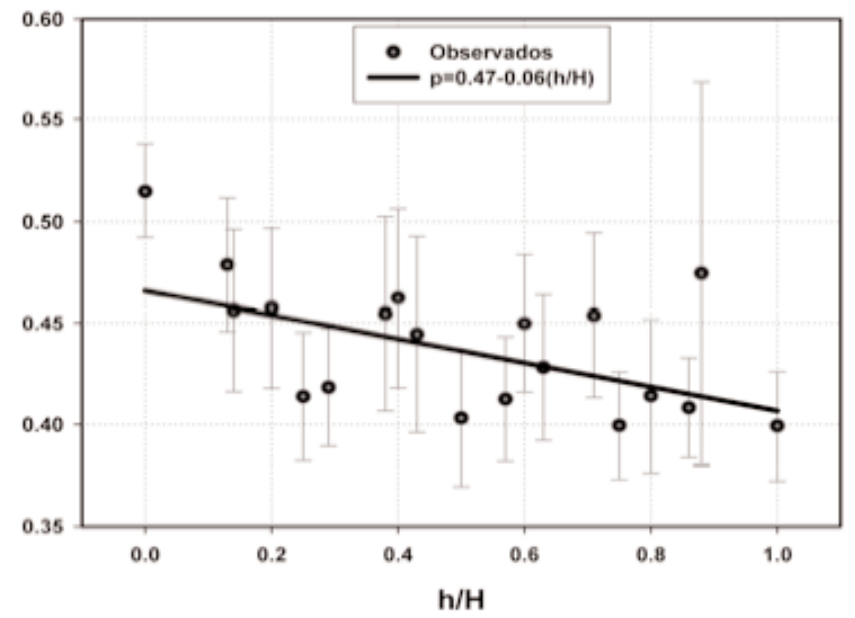

Figura 5. Relación entre la densidad promedio y la altura relativa para el duramen de especies de Pinus (P. arizonica, P. durangensis, P. engelmannii, P. teocote, P.cooperi y $P$. leiophylla) del norte del Estado de Durango, México. 


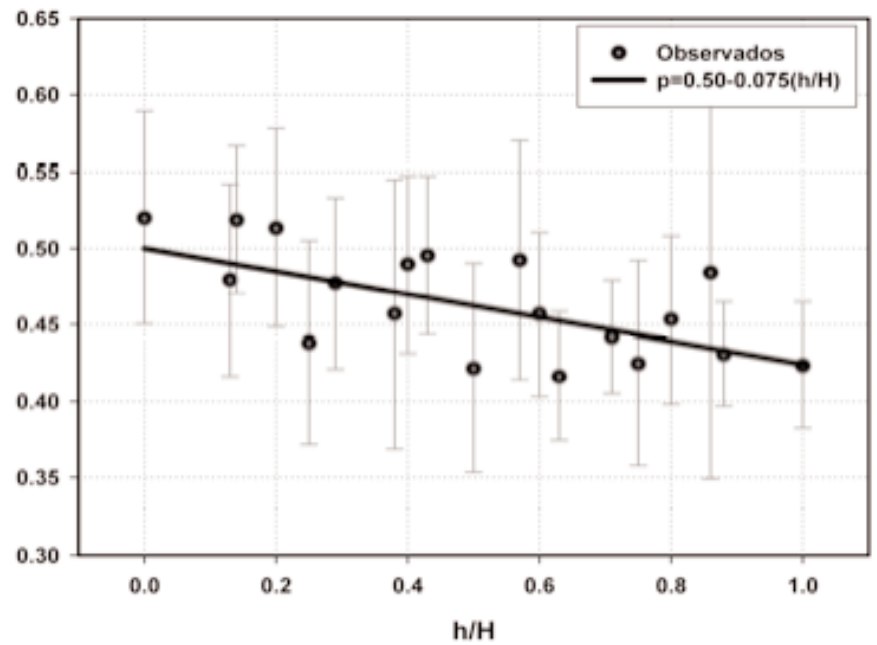

Figura 6. Relación entre la densidad promedio y la altura relativa para la albura de especies del género Pinus ( $P$. arizonica, $P$. durangensis, $P$. engelmannii, $P$. teocote,

P.cooperi y $P$. leiophylla) del norte del Estado de Durango, México.

2,25 (0.15); $3,37(0.22) ; 4,5(0,30)$ o 5,62 $(0,37)$ metros, respectivamente. Solo cuando los árboles tienen una altura de $6 \mathrm{~m}$, la probeta puede tomarse a $1,3 \mathrm{~m}$.

Las líneas rectas que describen la relación $\rho$ vs $\mathrm{h} / \mathrm{H}$ para la albura y el duramen en el género Pinus no se diferencian estadísticamente, de acuerdo con el análisis de covarianza $(P>F=0,58)$. Por esta razón, se deriva finalmente una sola ecuación, con la siguiente función matemática: $\rho=[0,487-0,0697(\mathrm{~h} / \mathrm{H})]$; $r^{2}=0,32 ; P=0,0001$. Esta ecuación posee interpretaciones físicas, 0,487 es la densidad básica promedio esperada para estas especies a la base del fuste. La pendiente negativa de la ecuación explica la reducción relativa de la densidad de la madera vieja en contraste con la madera nueva, así como también la proporción de albura y duramen. Es decir, a mayor altura del árbol, el contenido relativo de madera nueva y de albura son mayores que en la base del fuste.

\section{CONCLUSIONES}

El valor promedio encontrado en la densidad básica del género Pinus fue de $0,47 \mathrm{~g} \mathrm{~cm}^{-3} \pm 0,04 \mathrm{~g} \mathrm{~cm}^{-3}$, considerándose como una madera medianamente pesada, mientras que el valor promedio encontrado en el Quercus fue de $0,77 \mathrm{~g} \mathrm{~cm}^{-3} \pm$ $0,02 \mathrm{~g} \mathrm{~cm}^{-3}$, considerándose como una madera altamente pesada, de acuerdo con la clasificación vigente. En general, la densidad básica resultó ser estadísticamente igual entre los componentes albura y duramen, en los géneros Pinus y Quercus. Tampoco la altura relativa del árbol fue una fuente de variación importante en el género Quercus. Sin embargo, se encontraron diferencias estadísticas en la densidad básica entre los componentes de albura y duramen y la altura relativa de la troza en el género Pinus. Cuando se desarrolla una ecuación que define el cambio de la densidad básica con la altura relativa del árbol, se recomienda extraer las probetas para determinar este componente tecnológico a una 
altura relativa promedio con un intervalo de entre $0,21 \mathrm{H} \mathrm{m}$ a $0,24 \mathrm{H} \mathrm{m}$. Solo en árboles con $6 \mathrm{~m}$ de altura, la probeta se debe extraer a $1,3 \mathrm{~m}$.

\section{REFERENCIAS}

Chavé, J. 2002. Medición de densidad de madera en árboles tropicales. Manual de campo. PAN-AMAZONIA. $7 \mathrm{p}$.

Chavé, J., C. Andalo, S. Brown, M.A. Cairns, J.Q. Chambers, D. Eamus, H. Fölster, F. Fromard, N. Higuchi, T. Kira, J.P. Lescure, B.W. Nelson, H. Ogawa, H. Puig, B. Riéra y T. Yamakura. 2005. Tree allometry and improved estimation of carbon stocks and balance in tropical forests. Oecologia 145(1):87-99.

Daniel, T.W., J.A. Helms, y F.S. Baker. 1982. Principios de silvicultura. McGraw-Hill. México. 492 p.

Echenique-Manrique. R. y V. DíazGómez. 1972. Algunas características tecnológicas de la madera de 11 especies mexicanas. Bol. Téc. Num. 27. INIF. México. 71 p.

Echenique-Manrique. R. y R. Plumptre. 1994. Guía para el uso de maderas de México y Belice. Universidad de Guadalajara. Consejo Británico. Laboratorio de Ciencia y Tecnología de la Madera. Universidad de Oxford. 196 p. ISBN 968-895-517-5.

Fearnside, P.M. 1997. Wood density for estimating forest biomass in Brazilian Amazon. Forest Ecology and Management 90(1):59-89.

Hocker Jr., H.W. 1984. Introducción a la biología forestal. AGT Editor, S.A. México. 446 p.
INEGI (Instituto Nacional de Geografía, Estadística e Informática). 2002. Anuario Estadístico del Estado de Durango. Aguascalientes, México. $685 \mathrm{p}$.

Jenkins, J.C., R.A. Birdsey y Y. Pan. 2001. Biomass and NPP estimation for the mid-Atlantic region (USA) using plotlevel forest inventory data. Ecological Applications 11:1174-1193.

López L., M. y S. Valencia M. 2001. Variación de la densidad relativa de la madera de Pinus greggii Engelm. del norte de México. Madera y Bosques 7(1):37-46.

Miles, P.D. y W.B. Smith. 2009. Specific gravity and other properties of wood and bark for 156 tree species found in North America. USDA FS. Northern Research Station. Research Note NRS-38. Delware, OH. EUA.

Mohren, F. y K. Klein Goldewijkt. 1994. $\mathrm{CO}_{2}$ fix model. Institute of Forestry and Nature Research. Wageningen, Países Bajos.

Nájera, L., V. A. Zacarías, G. Méndez y L. Graciano. 2005. Propiedades físicas y mecánicas de la madera en Quercus laeta Liemb. de El Salto, Durango. Ra Ximhai, septiembrediciembre, vol. 1, núm. 3. Universidad Autónoma Indígena de México Mochicahui, El Fuerte, Sinaloa. p. 559-576.

Návar, J., N. González, y J. Graciano. 2005. Carbon sequestration by forest plantations of Durango Mexico. Madera y Bosques 11(2): 15-34.

Návar, J., 2009a. Allometric equations for tree species and carbon stocks for 
forests of northwestern Mexico. Forest Ecology and Management 257:427-434. doi:10.1016/j.foreco. 2008.09.028

Návar, J., 2009b. Biomass component equations for Latin American species and groups of species. Annals of Forest Science 66:208-216.

Návar, J. 2009c. Manual de Métodos Estadísticos. Facultad de Ciencias Forestales-UANL. Linares, N.L., México.

Návar, J. 2010. Methods of Assessment of Aboveground Tree Biomass. In: Biomass, M. Momba and Faizal Bux, (eds.). 202 p. InTech. Rijeka, Croatia.

Návar, J. 2011. Probabilidad y estadística aplicadas al manejo de recursos naturales y medio ambiente. McGraw Hill, México, D.F.

Parolin, P. 2002. Radial gradients in wood specific gravity in trees of Central Amazonian floodplains. IAWA Journal 23:449-457.
Pearson, R. y Williams, J. 1952. Review of methods for sampling of timber. Forest Products Journal 8(10): 263-268.

Pérez, O.F., R. Castro y O. Sadaaki. 2000. Índices de Calidad de Madera en Pinus taeda de Rivera para la optimización en el uso final. Proyecto de Tecnología de Ensayo de Productos Forestales LATU-JICA (1998-2003). Informe de Investigación núm. 2.

Van Buijtenen, J.P. 1963. Inheritance of wood properties and their relation to growth rate in Pinus taeda. World Consultation on Forest Genetics and Tree Improvement. FAO. Roma. 13 p.

Zobel, B.J. y J.P. Van Buijtenen. 1989. Word variation, its causes and control. Springer-Verlag. 363 p.

Zobel, B.J. y J.T. Talbert. 1988. Técnicas de mejoramiento genético de árboles forestales. Limusa. México. $545 \mathrm{p}$. 